\title{
Cervical cancer screening in Poland in current SARS-CoV-2 pandemic: Interim guidelines of the Polish Society of Gynecologists and Obstetricians and the Polish Society of Colposcopy and Cervical Pathophysiology - a summary January 2021
}

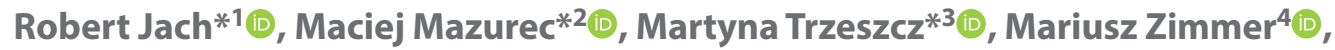 \\ Witold Kedzia ${ }^{5}$, Hubert Wolski6 ${ }^{6}$
}

${ }^{1}$ Division of Gynecologic Endocrinology, Jagiellonian University Medical College, Cracow, Poland

${ }^{2}$ Corfamed Women's Health Center, Wroclaw, Poland

${ }^{3}$ Division of Pathology and Clinical Cytology, University Hospital in Wroclaw, Poland

${ }^{4}$ Second Department of Gynecology and Obstetrics, Wroclaw Medical University, Poland

${ }^{5}$ Division of Developmental Gynecology and Sexuology, Department of Perinatology and Gynecology, Poznan University of Medical Sciences, Poland

${ }^{6}$ Division of Obstetrics and Gynecology, Poviat Hospital in Zakopane, Poland

${ }^{*}$ Authors should be regarded as the first authors due to the equal contribution to the article

\begin{abstract}
The Polish Society of Colposcopy and Cervical Pathophysiology (PTKiPSM) together with the Polish Society of Gynecologists and Obstetricians (PTGiP) issued a final summary of interim guidelines for secondary cervical cancer prevention during the SARS-CoV-2 pandemic based on the analysis of the latest directional publications and the authors' own experiences. The aim of the summary is to facilitate the implementation of the most effective possible screening of cervical precancerous lesions and cervical cancer due to temporary significant limitation of screening as a consequence of the ongoing epidemiological threat. These final guidelines are taking into account the 2020 call of the World Health Organization (WHO) for global epidemiological elimination of cervical cancer. The guidelines supplement the interim guidelines of PTKiPSM and PTGiP announced in March 2020 on the possible deferral of diagnostic and therapeutic procedures in patients with abnormal screening tests results in secondary prevention of cervical cancer in current pandemic.
\end{abstract}

Key words: cervical cancer prevention; abnormal screening results; HPV testing; cervical cytology; selfsampling; SARS-CoV-2 pandemic; guidelines

The recommendations present the current management which may be modified and changed in justified cases, after a thorough analysis of a given clinical situation, which in the future may constitute the basis for their modification and update.

Interim guidelines apply to the period indicated only.

\section{INTRODUCTION}

In May 2020, the World Health Organization (WHO) called on all institutions in the world involved in the cervical cancer prevention to take action to eliminate cervical cancer as a population problem by the end of the century, which is to reduce the number of cases to 
a level of a very rare disease ( $\leq 4$ cases/100,000/year). According to WHO, European countries should achieve this goal earlier, i.e. between 2055 and 2059. WHO has also defined goals to be reached in the short term, which will allow the achievement of the indicated main goal. The year 2030 is the time limit for all countries to meet the minimum targets defined by the abbreviation "90-70-90" which includes:

- covering $90 \%$ of girls by the age of 15 with the full vaccination against human papillomavirus (HPV),

- covering $70 \%$ of women with a highly effective screening test at least twice during their lifetime, in 35 and again in 45 years of age,

- covering $90 \%$ of women with diagnosis of precancerous lesions or cervical cancer with appropriate treatment and care [1-3].

The European response to WHO's call is a joint initiative of the European CanCer Organization (ECCO), the European Society of Gynecological Oncology (ESGO) and the European Federation for Colposcopy (E.F.C.). The European proposals for goals that should be achieved by 2030 have a wider scope and have been specified in relation to the WHO base document and include, among others:

- covering $90 \%$ of girls and boys with the full vaccination against HPV by the age of 15 ,

- covering $70 \%$ of women in Europe at the indicated age by cervical cancer screening using a clinically validated test for high-risk human papillomavirus types (HRHPV) over the last five years,

- providing $90 \%$ of women with diagnosis of histologic histological high-grade squamous intraepithelial lesions (HSIL) with subcategorization to CIN 3 and higher (CIN $3+$ ) with treatment within 3 months from diagnosis, and all women diagnosed with cervical cancer ensure access to appropriate oncological care, including palliative care.

The document also points to the uniqueness of the challenges related to the SARS-CoV-2 pandemic, including the need for European women to access, especially during the pandemic, HRHPV self-sampling kits [4].

The Polish response to the WHO's call is the COLPOSCOPY 2020 Project with already published $[5,6]$ and planned for publication guidelines for secondary cervical cancer prevention in Poland.

The achievement of the goals set by WHO is obviously hindered by the SARS-CoV-2 pandemic that has significantly inhibited or limited secondary cancer prevention, including cervical cancer. American data indicate a reduction of up to $94 \%$ in the number of cervical cancer screening tests performed between week 10 and week 20 of this year compared to previous years. In June, the situation improved slightly, setting the screening tests deficit at $67 \%$, which is still a very high-level [7]. Polish data for 2020 in the secondary cervical cancer prevention financed from public funds are highly worrying $-13.84 \%$ of the planned female population was screened [8]. Data for cervical cancer screening in the opportunistic model, apart from public funding, are not known.

Interim guidelines related to the pandemic are the reaction of scientific societies to the rapidly changing screening reality in this period, which could not be foreseen in the planned model of secondary cervical cancer prevention in Poland. That consequently means the need to implement modifications and updates depending on the changing external epidemiological situation [9].

\section{RECOMMENDED CERVICAL CANCER SCREENING APPROACH DURING THE SARS-COV-2 PANDEMIC}

The long-term consequences of limiting or discontinuing the population-based cervical cancer secondary prevention tools can be difficult to predict and control. In the persistent SARS-CoV-2 pandemic and its unforeseeable course and completion date, taking into account the WHO's May 2020 call for action on cervical cancer prevention and its goals, the following screening approach is temporarily recommended in Polish conditions during the epidemiological threat associated with the SARS-CoV-2 pandemic. The overriding immediate goal of these guidelines is to achieve in Poland in the time of a pandemic the highest possible share of the population covered by screening, optimally according to the recommended interim screening models, with the most effective diagnostic possible triage of patients requiring referral to colposcopy.

The recommended cervical cancer screening in Poland during the SARS-CoV-2 pandemic includes the following strategies:

1. In the case of an epidemiological situation that allows the collection of material from the cervix by a gynecologist or midwife and the availability of dedicated diagnostic-laboratory facilities, liquid-based screening (LBS), i.e., the use of a liquid preparation as a carrier of the collected cell material, is recommended. LBS is a method that enables the performance of several diagnostic tests from one collection, reduces the number of necessary visits to one in precolposcopy stage and eliminates the use of aerosol fixer.

Liquid-based screening should only use clinically validated tools: liquid media, molecular tests for 14 high-risk human papillomavirus types (HRHPV14), separately for the primary HRHPV14 test and for the cotesting [10], and for the immunocytochemical test $\mathrm{p} 16 / \mathrm{Ki} 67$ as well [11]. Until validated diagnostic tools are approved for Polish population, it has been temporarily accepted to rely on the appropriate registrations of the American Food and Drug Administration (FDA), and additionally on the VALGENT (Validation of HPV 
Genotyping Tests) and Meijer's protocols for HRHPV14 tests $[12,13,14]$

For liquid-based screening, an implementation of the HPV-based model is recommended, i.e.:

- $\quad$ primary test for HRHPV14

- or cotesting which includes HRHPV14 testing and liquid-based cytology (LBC), with further diagnostic-therapeutic management depending on tests results and, in indicated cases, including a past history.

Clinical management based on the HSIL (CIN 3+) risk assessment calculated using the current tests results and screening history, presented in the ASCCP 2019 (American Society for Colposcopy and Cervical Pathology) recommendations, is temporarily acceptable approach in the diagnostic-therapeutic management in Poland. The screening history was defined as recent past tests (in the last 5 years) and/or treatment for histological HSIL with sub-categorization to CIN 2 and higher (CIN 2+) in the previous 25 years $[10,15,16]$. Presented management is applicable only when screening tests approved by the FDA are used.

All HRHPV14 positive cases require further diagnostic procedures using the histologic HSIL-risk triage test (CIN $2+$ ), i.e., cytology or $\mathrm{p} 16 / \mathrm{Ki} 67$ test. For HPV positive women, ICC p16/Ki67 testing should be regarded as an alternative reflex test to cytology and as a triage test for abnormal cytology result. A laboratory preparation of p16/Ki67 test should be carried out using fully automated system only and a morphologic evaluation should be performed by trained cytopathologist - both are obligatory FDA conditions for p16/Ki67 testing [11].

An optional management in the time of a pandemic is referring to immediate colposcopy all patients with detected HRHPV types 16 and/or 18, and in the cases of using the molecular testing with extended genotyping beyond types 16 and 18 [HPV extended genotyping (HPV-xGT)], referring to immediate colposcopy patients with detected HPV type 31 it is optionally also recommended $[17,18]$.

Currently, the use of methylation-based methods does not have sufficient clinical validation in cervical cancer screening [19].

Four basic interim HPV-based screening models are recommended for Polish conditions:

- $\quad$ screening model 1 - primary HRHPV14 testing

$-1 \mathrm{~A}-$ with reflex cytology

- 1B - with reflex p16/Ki67 test

- screening model 2 - primary cotesting (HRHPV14+LBC)

$-2 \mathrm{~A}$ - without reflex testing

- 2B - with reflex $\mathrm{p} 16 / \mathrm{Ki} 67$ test

- screening model 3 - primary HRHPV14 extended genotyping (in patients with previously unknown HRHPV14 status) with reflex cytology
- screening model 4 - primary HRHPV14 testing based on the ASCCP 2019 recommendations [10, 15, 16]; (ASCCP 2019 Recommendations in Polish: Medycyna Praktyczna Ginekologia i Położnictwo No. 05 and No. 06/2020)

The detailed recommended management strategies for screening models 1-3 are shown in Figures 1-7 (see page 169-172).

Due to the lack of obligatory reporting for the required assessment and quality control parameters for liquid-based cytology in Poland, including in particular, the percentage of individual cytological diagnoses compared to the values reported by the College of American Pathologists (CAP), cyto-virological correlations (CVC) and cyto-histological correlations $(\mathrm{CHC})$, it is recommended to report minimally the parameters indicated in the relevant recommendations of the COLPOSCOPY 2020 Project (paper in progress).

2. Self-sampling, defined as a self-collection of vaginal material by the patient with the use of a dedicated brush, is recommended in the primary HPV-based screening as the basic tool for cervical cancer screening for cases when a social distance is necessary and/or concerns of secondary cervical cancer prevention recipients related to risk of infection with SARS-CoV-2 virus occur, which in its extreme form leads to leave of screening [20-22].

Due to the lack of sufficiently validated HRHPV14 tests in Poland for the self-sampling method, in the cases of positive HPV result obtained with this method, a routine material sampling from the cervix is necessary (an appointment and a contact with medical personnel is needed) to confirm the positive result on the validated molecular HRHPV testing. In the cases of a confirmed positive HRHPV result by validated HPV testing, further management is described in point No. 1.

All HRHPV14 negative tests results should be confirmed with the currently recommended HRHPV14 tests collected by qualified medical personnel or with the use of a self-sampling testing approved for Polish population.

3. The self-sampling method is also recommended as a triage tool enabling the reduction of social contacts for the continuation of secondary cervical cancer screening for minor cytological abnormalities [atypical squamous cells of undetermined significance (ASC-US) or low-grade squamous intraepithelial lesions (LSIL)], according to Polish recommendations 2016 [23]. It is applicable, when the original liquid-based cervical material obtained during a routine cervical screening is not available in the laboratory.

4. In the case of an epidemiological situation allowing the cervical sampling by a doctor or midwife, and when 
a performing liquid-based preparation in not possible, a conventional cytology is acceptable screening strategy in opportunistic models from public and private funds, with further management depending on cytology results and the availability of diagnostic tests, based on the current recommendations [23-25].

It is emphasized that this screening strategy requires the patient's next appointment, especially in the cases of minor cytological abnormalities (ASC-US or LSIL) or in the cases of unsatisfactory cytology results, which should be considered as suboptimal during a pandemic.

5. Expanding a participation of non-gynecologists (general practitioners, midwifes and community nurses) in secondary cervical cancer prevention, particularly in a promoting and education in self-sampling method is recommended, as an effective screening alternative tool to sampling performed by medical personnel, when patient is unable to participate in a routine cervical cancer screening, and/or the strict national or regional epidemiological cautions are introduced.

\section{INTERIM INDICATIONS FOR COLPOSCOPY}

The following results are recommended as decision parameters in the referral of the patient to colposcopy with biopsy within 3 months, regardless of the other tests results: - positive HRHPV 16 and/or 18 and/or 31 type (HPV type 31 when using the HPV-xGT testing) in the cases of unknown patient's HRHPV status within the last 3 years, - positive HRHPV 16 and/or 18 and/or 31 type (HPV type 31 when using the HPV-xGT test) detected again after 12 months in the cases of a patient previously monitored,

- positive HRHPV N16/N18 (one or more) or HRHPV N16/N18/N31 (one or more) (N16/N18/N31 type in cases of using the HPV-xGT testing) detected again after 12 months in the cases of a patient previously monitored, - positive p16/Ki67 test result,

- atypical squamous cells, cannot exclude high-grade squamous intraepithelial lesion (ASC-H), HSIL or atypical glandular cells (AGC) cytology results.

If an invasive process is suspected, further diagnostic-therapeutic management must be undertaken immediately.

The possibility of deferral the diagnostic-therapeutic management is maintained as allowed, based on the PTGiP and PTKiPSM Interim Guidelines of March 22, 2020, with the update of May 2, 2020 [7].

\section{SUPPORTING ACTIONS}

An effective and quick education of medical doctors (gynecologists, general practitioners, pathologists), laboratory diagnosticians, community midwives, community nurses and other medical workers is recommended as necessary for the effective implementation of these interim guidelines for secondary cervical cancer prevention in Poland in the SARS-CoV-2 pandemic, with a key role of PTKiPSM and support of the Cervical Pathology, Colposcopy and Cytology Subdivision of PTGiP.

Scientific societies (PTGiP and PTKiPSM) call for an adequate response to the pandemic all of institutions responsible for secondary cervical cancer prevention financed from public funds in Poland, taking into account the presented guidelines and the actual epidemiological situation.

The authors of these guidelines emphasize - in line with the position of opinion-forming European and American directional authorities $[4,10]$, the need to use only clinically validated liquid-based media in cervical cancer screening and HRHPV14 molecular tests, with a dedicated specific liquid-based laboratory preparation, independently for LBS and self-sampling [10, 21]. This also applies to all recent past screening tests, if included in a risk stratification.

The tests registered by the FDA for cervical cancer screening using the primary HRHPV14 testing or cotesting and screening tests validated with VALGENT or Meijer's protocols are tests of temporary choice for use in Poland due to the lack of Polish clinical validation for any of these screening tests (an organization of tests' validation in the progress). Similarly, when the p16/Ki67 immunocytochemical test is used, only automated preparation is recommended with evaluation by directionally trained cytopathologist to employ the FDA registration [11].

Regarding the selfsampling method, until the tests are validated for Polish conditions, it is temporarily recommended to take advantage of Australia and Netherlands expertise in support cervical cancer screening by this method. The authors of the guidelines also highlight the need to use validated tools to collect material from the cervix and/or vagina, also in the cases of the self-sampling method [21].

For the colposcopy, the need of its standardization and algorithmization according to the colposcopic protocols guidelines and the use of Polish colposcopic nomenclature in accordance with the COLPOSCOPY 2020 Project is pointed $[5,6]$.

Figures 1-7 describing recommended HPV-based screening models depending on the primary screening tests results are presented below. 


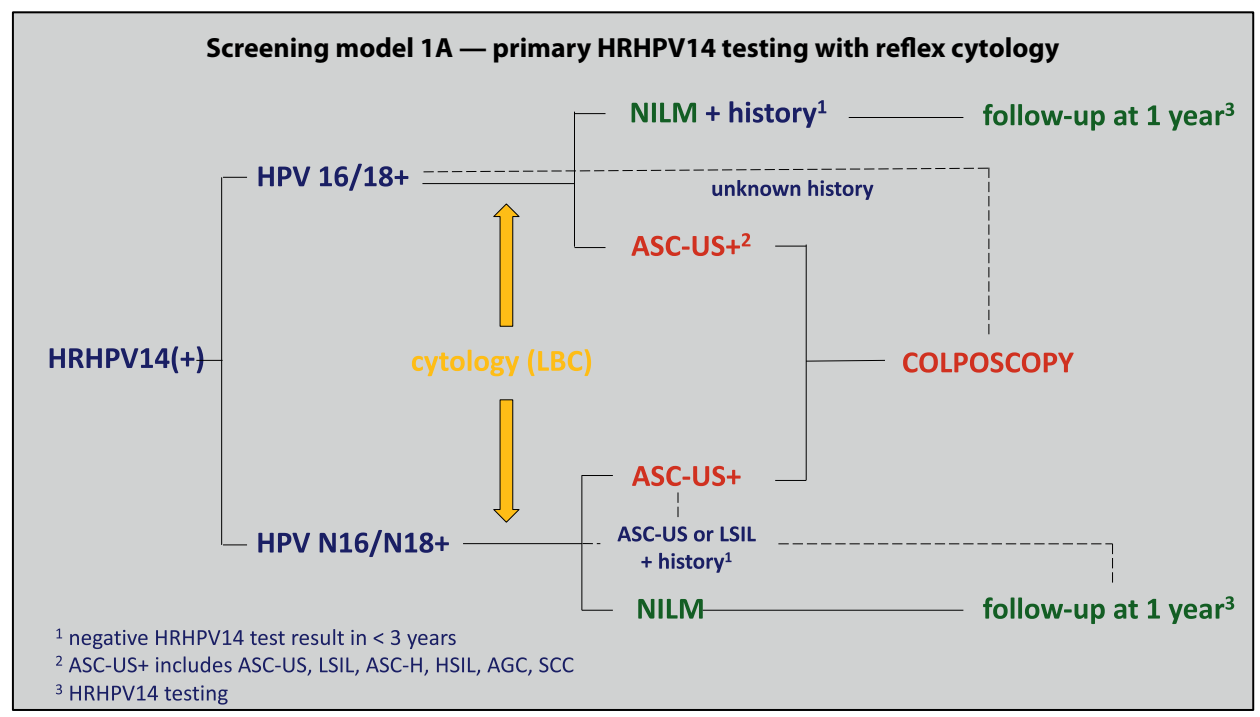

Figure 1. Management algorithm for positive primary 14 types of high-risk human papillomavirus test results with reflex cytology; HRHPV14 - 14 types of high-risk human papillomavirus; NILM - negative for intraepithelial lesion or malignancy; ASC-US+ - atypical squamous cells of undetermined significance and higher cytological abnormalities; HPV — human papillomavirus; LBC — liquid-based cytology; ASC-US — atypical squamous cells of undetermined significance; LSIL — low-grade squamous intraepithelial lesions; ASC-H — atypical squamous cells, cannot exclude high-grade squamous intraepithelial lesion; HSIL — high-grade squamous intraepithelial lesions; AGC — atypical glandular cells; SCC - squamous cell carcinoma

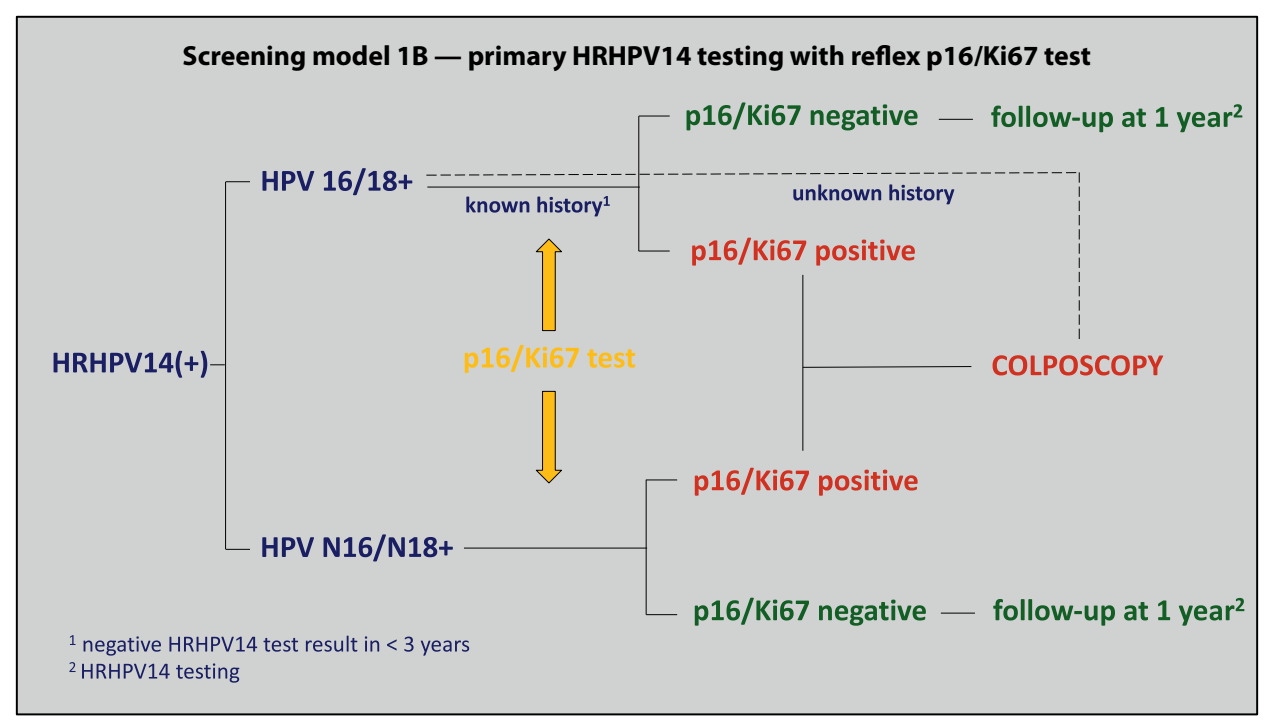

Figure 2. Management algorithm for the positive primary 14 types of high-risk human papillomavirus test results with reflex p16/Ki67 double immunocytochemical test; HRHPV14 - 14 types of high-risk human papillomavirus; HPV — human papillomavirus; 


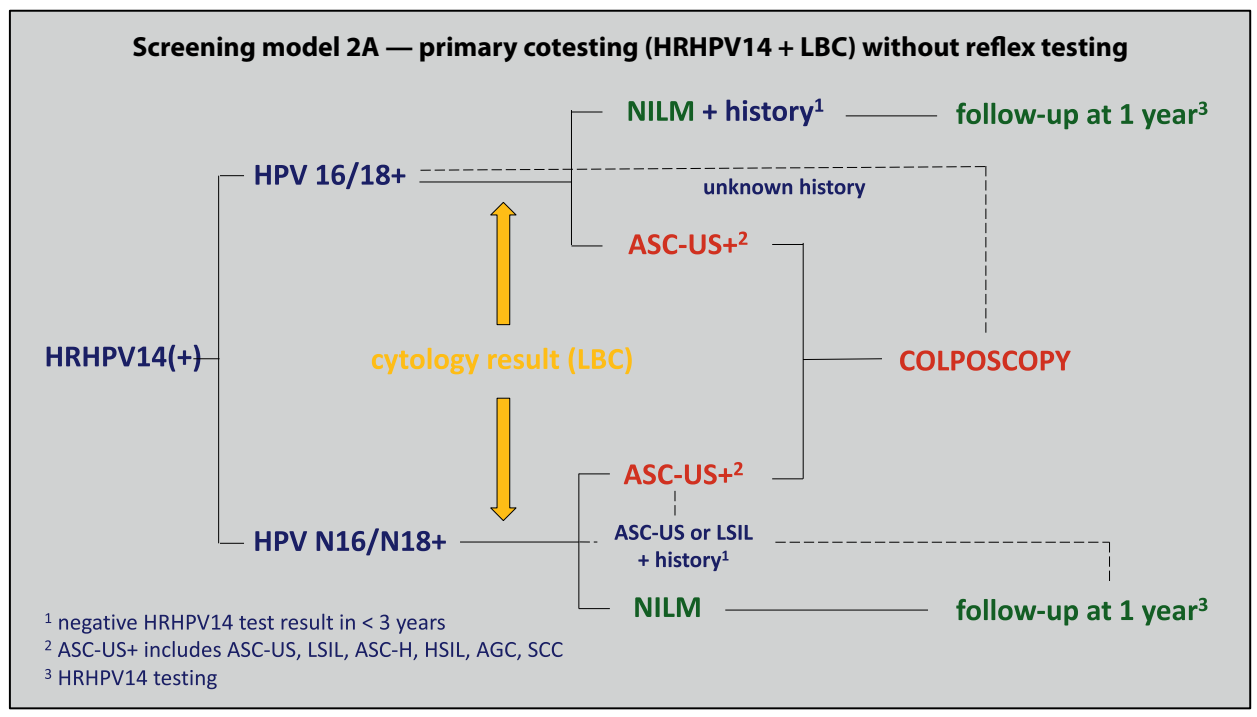

Figure 3. Management algorithm for the cotesting in cases of a positive 14 types of high-risk human papillomavirus test result; HRHPV14 - 14 types of high-risk human papillomavirus; LBC — liquid-based cytology; NILM - negative for intraepithelial lesion or malignancy; ASC-US+ — atypical squamous cells of undetermined significance and higher cytological abnormalities; HPV — human papillomavirus; ASC-US — atypical squamous cells of undetermined significance; LSIL — low-grade squamous intraepithelial lesions; ASC-H — atypical squamous cells, cannot exclude high-grade squamous intraepithelial lesion; HSIL — high-grade squamous intraepithelial lesions; AGC — atypical glandular cells; SCC - squamous cell carcinoma

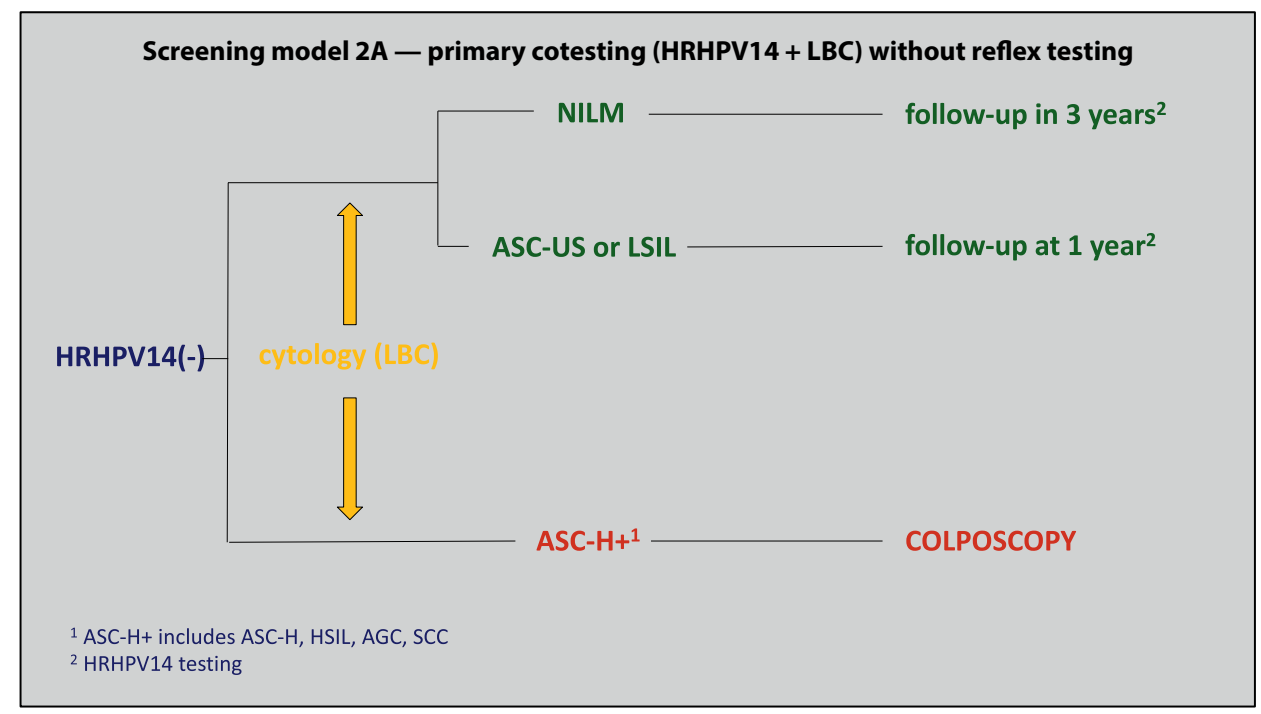

Figure 4. Management algorithm for the cotesting in cases of negative 14 types of high-risk human papillomavirus test result; HRHPV14 - 14 types of high-risk human papillomavirus; LBC — liquid-based cytology; NILM — negative for intraepithelial lesion or malignancy; ASC-US - atypical squamous cells of undetermined significance; LSIL — low-grade squamous intraepithelial lesions; ASC-H — atypical squamous cells, cannot exclude high-grade squamous intraepithelial lesion; HSIL — high-grade squamous intraepithelial lesions; AGC — atypical glandular cells; SCC - squamous cell carcinoma 


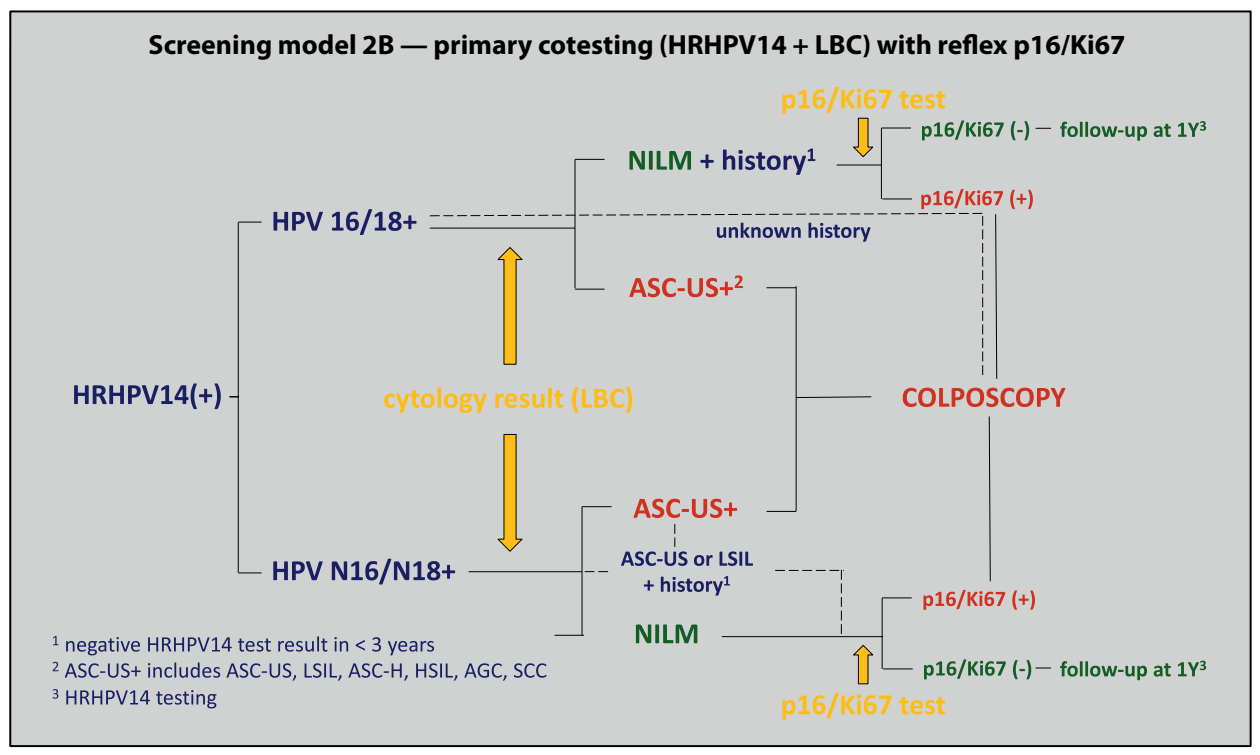

Figure 5. Management algorithm for the positive 14 types of high-risk human papillomavirus test results obtained in cotesting with reflex p16/Ki67 double immunocytochemical test; HRHPV14 - 14 types of high-risk human papillomavirus; HPV — human papillomavirus; LBC - liquid-based cytology; NILM — negative for intraepithelial lesion or malignancy; ASC-US+ - atypical squamous cells of undetermined significance and higher cytological abnormalities; ASC-US — atypical squamous cells of undetermined significance; LSIL — low-grade squamous intraepithelial lesions; ASC-H — atypical squamous cells, cannot exclude high-grade squamous intraepithelial lesion; HSIL — high-grade squamous intraepithelial lesions; AGC — atypical glandular cells; SCC — squamous cell carcinoma

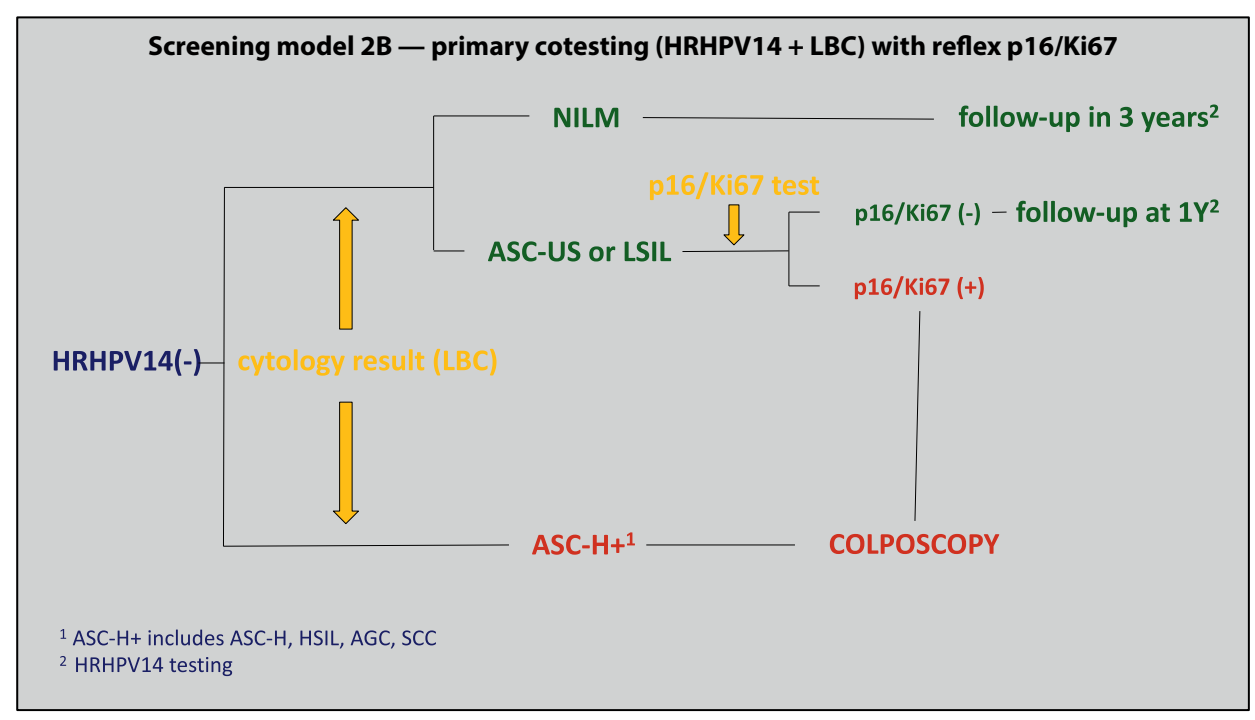

Figure 6. Management algorithm for the negative 14 types of high-risk human papillomavirus test results obtained in cotesting with reflex p16/Ki67 double immunocytochemical test; HRHPV14 - 14 types of high-risk human papillomavirus; LBC — liquid-based cytology; NILM — negative for intraepithelial lesion or malignancy; ASC-US — atypical squamous cells of undetermined significance; LSIL — low-grade squamous intraepithelial lesions; ASC-H — atypical squamous cells, cannot exclude high-grade squamous intraepithelial lesion; HSIL — high-grade squamous intraepithelial lesions; AGC — atypical glandular cells; SCC — squamous cell carcinoma 


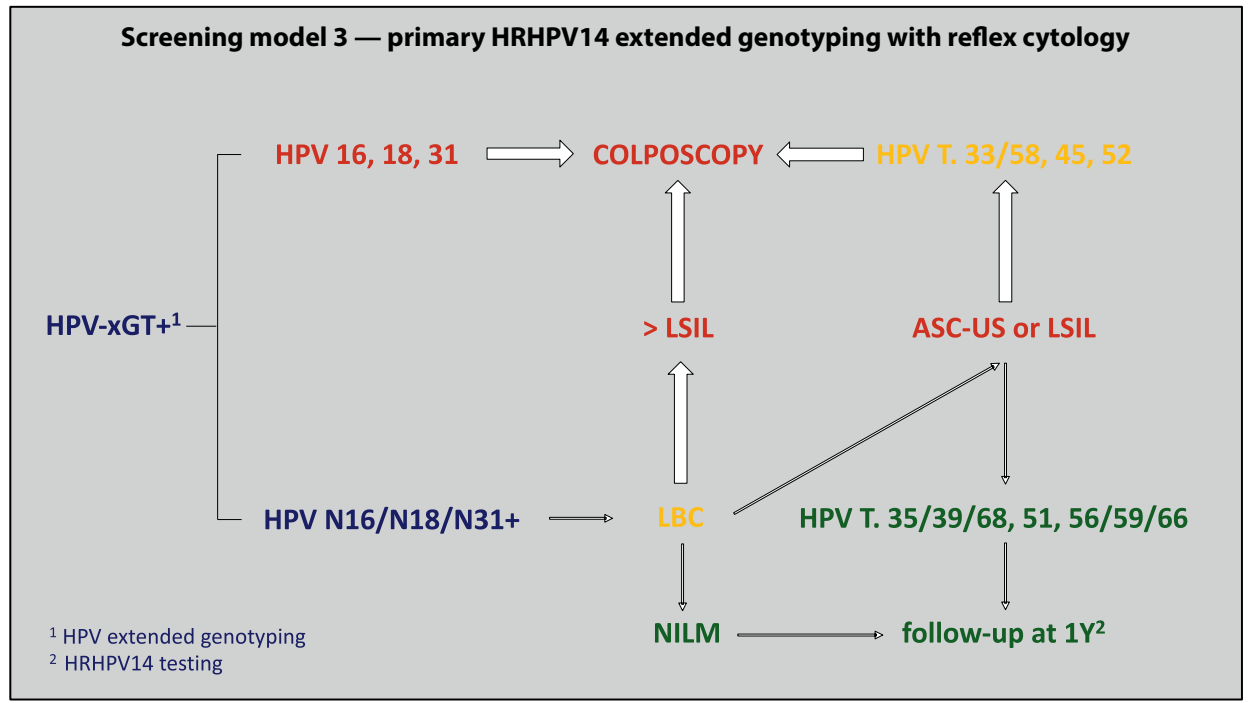

Figure 7. Management algorithm for the primary 14 types of high-risk human papillomavirus extended genotyping results with reflex cytology; HPV-xGT - human papillomavirus extended genotyping; HPV — human papillomavirus; LSIL — low-grade squamous intraepithelial lesions; LBC — liquid-based cytology; NILM — negative for intraepithelial lesion or malignancy; ASC-US — atypical squamous cells of undetermined significance

The above guidelines are not the final diagnostic management approach for abnormal screening tests results in secondary cervical cancer prevention.

They are not a substitute for a complete clinical evaluation of an individual case.

They should always be analyzed in the context of optimizing the diagnostic management in the patient's health interest.

They may be modified depending on changing conditions.

\section{REFERENCES}

1. WHO Call. Global Strategy Towards the Elimination of Cervical Cancer as a Public Health Problem. Geneva, Switzerland:WHO; 2019. https://www. who.int/docs/default-source/documents/cervical-cancer-eliminationdraft-strategy.pdf (05.06.2020).

2. Draft Global Strategy towards eliminating cervical cancer as a public health problem. WHO 5 April 2020/Publication. https://www.who. int/publications/m/item/draft-global-strategy-towards-eliminating-cervical-cancer-as-a-public-health-problem (05.06.2020).

3. Gultekin M, Ramirez PT, Broutet N, et al. World Health Organization call for action to eliminate cervical cancer globally. Int J Gynecol Cancer. 2020; 30(4): 426-427, doi: 10.1136/ijgc-2020-001285, indexed in Pubmed: 32122950 .

4. Arbyn M, Gultekin M, Morice $P$, et al. The European response to the WHO call to eliminate cervical cancer as a public health problem. Int J Cancer. 2021; 148(2): 277-284, doi: 10.1002/ijc.33189, indexed in Pubmed: 32638362.

5. Jach R, Mazurec M, Nowakowski A, Pityński K. KOLPOSKOPIA 2020: POLSKA NOMENKLATURA KOLPOSKOPOWA w oparciu o mianownictwo IFCPC 2011. Podsumowanie Konsensusu Ekspertów. Rekomendacje Grupy Ekspertów Polskiego Towarzystwa Ginekologów i Położników oraz Polskiego Towarzystwa Kolposkopii i Patofizjologii Szyjki Macicy. https:// www.ptgin.pl/sites/default/files/page-2020/NOMENKLATURA\%20KOLPOSKOPOWA\%20strona\%20www\%20.pdf (03.10.2020).

6. Jach R, Mazurec M, Trzeszcz M, et al. COLPOSCOPY 2020 - COLPOSCOPY PROTOCOLS: A Summary of the Clinical Experts Consensus Guidelines of the Polish Society of Colposcopy and Cervical Pathophysiology and the Polish Society of Gynaecologists and Obstetricians. Ginekol Pol.
2020; 91(6): 362371, doi: 10.5603/GP.2020.0075, indexed in Pubmed: 32627158.

7. Mast Ch, del Rio AM. Data Analysis | Update Delayed Cancer Screenings-A Second Look Abstract: Mid-June volumes of preventive breast, colon, and cervical cancer screenings remain $29 \%-36 \%$ lower than their pre-COVID-19 levels. Epic Health Research Network. July 17, 2020. https://ehrn.org/articles/delayed-cancer-screenings-a-second-look (03.10.2020).

8. NFZ. Dane o realizacji programów/Programy profilaktyczne. Według stanu na dzień 1 stycznia 2021 r. http://www.nfz.gov.pl/dla-pacjenta/programy-profilaktyczne/dane-o-realizacji-programow/ (06.01.2021).

9. Jach R, Mazurec M, Trzeszcz M, et al. Possible deferral of diagnostic and therapeutic procedures for patients with abnormal screening tests results in cervical cancer secondary prevention in current SARS-CoV-2 pandemic Interim guidelines of the Polish Society of Gynecologists and Obstetricians and the Polish Society of Colposcopy and Cervical Pathophysiology. Ginekol Pol. 2020; 91(7):428-431, doi: 10.5603/GP.2020.0103, indexed in Pubmed: 32779165.

10. Perkins RB, Guido RS, Castle PE, et al. 2019 ASCCP Risk-Based Management Consensus Guidelines Committee. 2019 ASCCP Risk-Based Management Consensus Guidelines for Abnormal Cervical Cancer Screening Tests and Cancer Precursors. J Low Genit Tract Dis. 2020; 24(2): 102-131, doi: 10.1097/LGT.0000000000000525, indexed in Pubmed: 32243307.

11. SUMMARY OF SAFETY AND EFFECTIVENESS DATA (SSED) CINTeC PLUS Cytology. Premarket Approval Application (PMA) Number: P190024. Date of FDA Notice of Approval: March 10, 2020. https://www.accessdata.fda. gov/cdrh_docs/pdf19/P190024B.pdf (05.06.2020).

12. FDA Executive Summary: New Approaches in the Evaluation for High-Risk Human Papillomavirus Nucleic Acid Detection Devices. https:// www.fda.gov/media/122799/download (10.10.2020).

13. Arbyn M, Snijders PJF, Meijer CJ, et al. Which high-risk HPV assays fulfi criteria for use in primary cervical cancer screening? Clin Microbiol Infect. 2015; 21(9): 817-826, doi: 10.1016/j.cmi.2015.04.015, indexed in Pubmed: 25936581.

14. Arbyn M, Hillermanns P. HPV assays validated for primary cervical cancer screening. https://www.hpvworld.com/articles/hpv-assays-validated-for-primary-cervical-cancer-screening/ (10.10.2020).

15. Egemen D, Cheung LiC, Chen X, et al. Risk Estimates Supporting the 2019 ASCCP Risk-Based Management Consensus Guidelines. J Low Genit Tract Dis. 2020; 24(2): 132-143, doi: 10.1097/LGT.0000000000000529, indexed in Pubmed: 32243308.

16. Demarco M, Egemen D, Raine-Bennett TR, et al. A Study of Partial Human Papillomavirus Genotyping in Support of the 2019 ASCCP Risk-Based Management Consensus Guidelines. J Low Genit Tract 
Dis. 2020; 24(2): 144-147, doi: 10.1097/LGT.0000000000000530, indexed in Pubmed: 32243309.

17. Bonde JH, Sandri MT, Gary DS, et al. Clinical Utility of Human Papillomavirus Genotyping in Cervical Cancer Screening: A Systematic Review. J Low Genit Tract Dis. 2020; 24(1): 1-13, doi: 10.1097/LGT.0000000000000494, indexed in Pubmed: 31714325.

18. Bonde J, Bottari F, Parvu V, et al. Bayesian analysis of baseline risk of CIN2 and $\geq$ CIN3 by HPV genotype in a European referral cohort. Int J Cancer. 2019; 145(4): 1033-1041, doi: 10.1002/ijc.32291, indexed in Pubmed: 30895602.

19. Cuschieri K, Ronco G, Lorincz A, et al. Eurogin roadmap 2017:Triage strategies for the management of HPV-positive women in cervical screening programs. Int J Cancer. 2018; 143(4): 735-745, doi: 10.1002/ijc.31261, indexed in Pubmed: 29341110.

20. Ajenifuja KO, Belinson J, Goldstein A, et al. Designing low-cost, accurate cervical screening strategies that take into account COVID-19: a role for self-sampled HPV typing2. Infect Agent Cancer. 2020; 15: 61, doi: 10.1186/s13027-020-00325-4, indexed in Pubmed: 33072179.

21. Hawkes D, Keung MHT, Huang Y, et al. Self-Collection for Cervical Screening Programs: From Research to Reality. Cancers (Basel). 2020; 12(4), doi: 10.3390/cancers12041053, indexed in Pubmed: 32344565.
22. Arbyn M, Smith SB, Temin S, et al. Collaboration on Self-Sampling and HPV Testing. Detecting cervical precancer and reaching underscreened women by using HPV testing on self samples: updated meta-analyses. BMJ. 2018; 363: k4823, doi: 10.1136/bmj.k4823, indexed in Pubmed: 30518635.

23. Nasierowska-Guttmejer A, Kędzia W, Wojtylak S, et al. Polish recommendations regarding diagnostics and treatment of cervical squamous intraepithelial lesions according to the CAP/ASCCP guidelines. Ginekol Pol. 2016; 87(9): 670-676, doi: 10.5603/GP.2016.0066, indexed in Pubmed: 27723078.

24. Józefiak A, Kędzia W, Kotarski J, et al. Guidelines for application of molecular tests identyfying HR HPV DNA in the prevention of cervical cancer. Statement of experts from PGS (PTG) and NCLD (KIDL). Ginekol Pol. 2013; 84: 395-399.

25. National Cervical Cancer Screening Programme in Poland Coordinating Centre, Polish Gynaecological Society, Polish Society of Pathologists, Polish Society of Colposcopy and Uterine Cervix Pathology. Management of abnormal pap smear--consensus guidelines of the National Cervical Cancer Screening Programme in Poland Coordinating Centre, the Polish Gynaecological Society, the Polish Society of Pathologists, and Polish Society of Colposcopy and Uterine Cervix Pathology. Ginekol Pol. 2009; 80(2): 129-138. 